\title{
THE HAND
}

\author{
D W Hodgkinson, N Kurdy, D A Nicholson, P A Driscoll
}

\section{Important anatomy}

Injuries to the hand and wrist account for about $15 \%$ of attendances at accident and emergency departments. The hand and wrist are complex structures and injury to just one small component can result in appreciable loss of function. Fortunately if the injury is identified early and managed appropriately function can be fully restored.
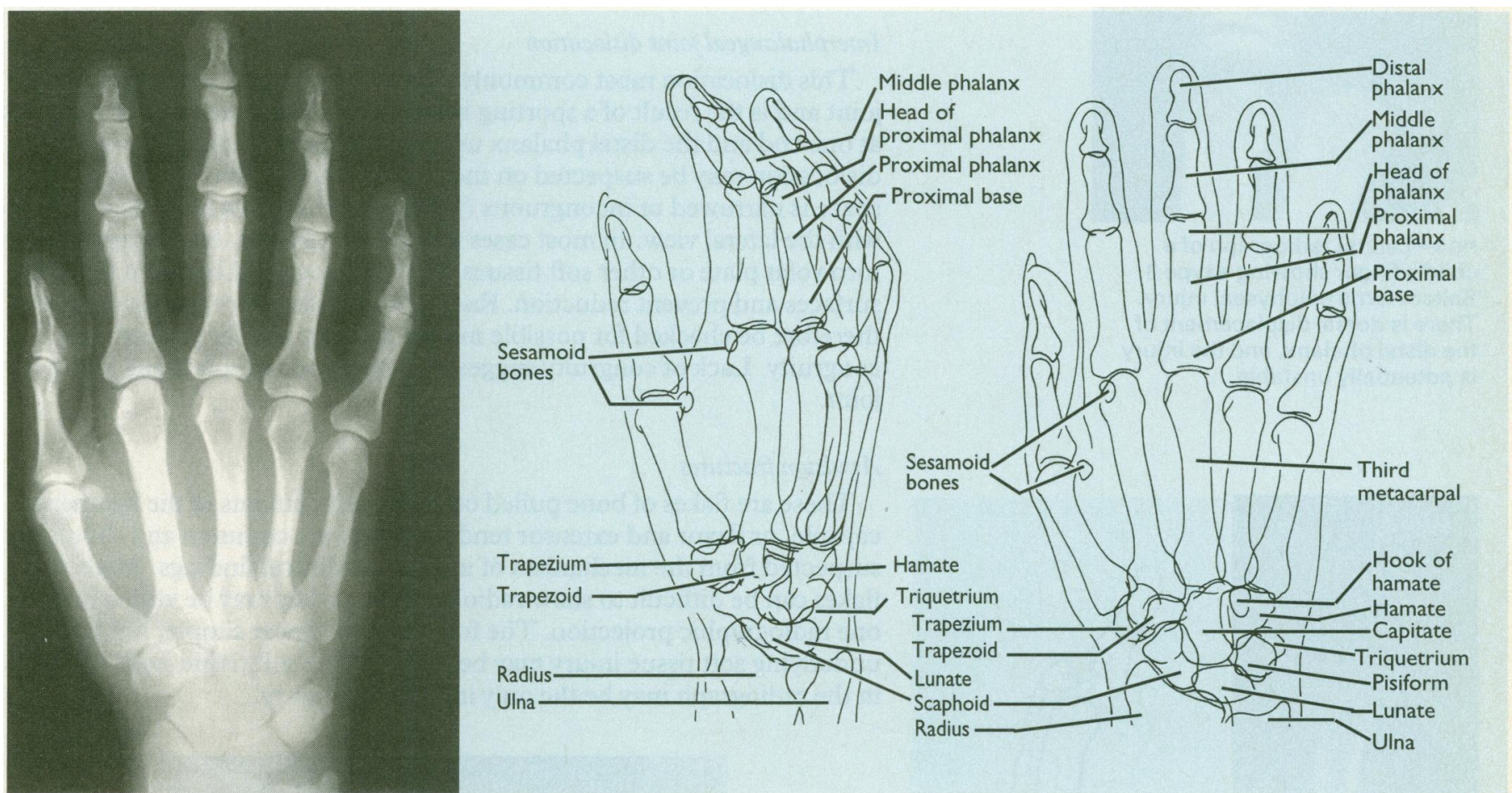

FIG 1-Posteroanterior radiograph and line drawing of normal hand.

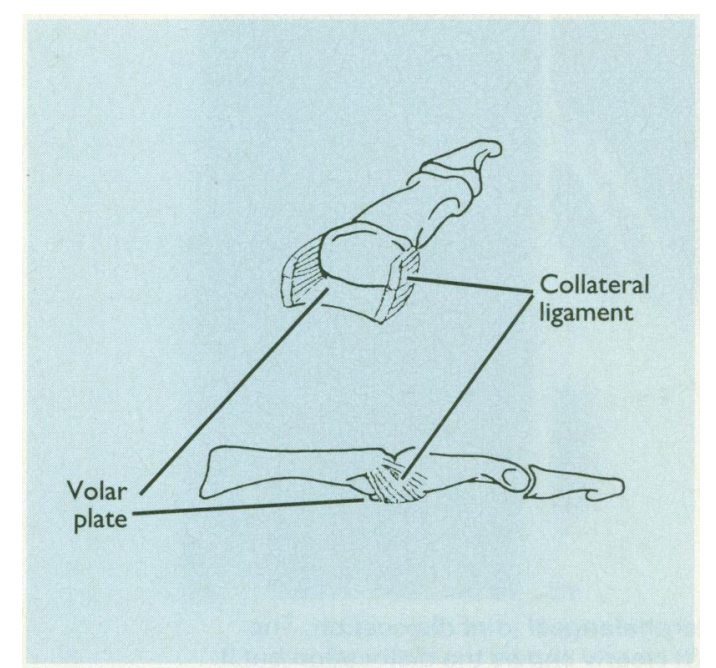

FIG 2-Collateral ligaments and volar plate of the proximal interphalangeal joint.
The 14 phalanges and five metacarpals in each hand consist of a head, a body, and a base. Collateral ligaments extend from the lateral margins of the head of each metacarpal bone and phalanx (except the terminal phalanx) across the appropriate joint space to insert on the lateral margin of the base of the apposing phalanx. The volar aspect of the interphalangeal and metacarpophalangeal joint capsule is thickened and forms a dense fibrous structure called the volar plate. Sesamoid bones may be found on the palmar surface of the hand. The two commonest are in the tendons of the thumb in the two heads of the flexor pollicis brevis at the metacarpophalangeal joint (fig 1). The other sesamoid bones of the hand are usually found at the metacarpophalangeal portion of the volar plate. Sideways movement of the thumb metacarpophalangeal joint is prevented by strong ulnar and radial collateral ligaments (fig 9).

\section{Development}

Secondary ossification centres (epiphyses) in the metacarpals and phalanges of the hand appear at the age of 2-3 years, and the growth plate normally closes at puberty. Skeletal age up to puberty can be judged accurately from hand and wrist radiographs as the sequence of development is age specific. 


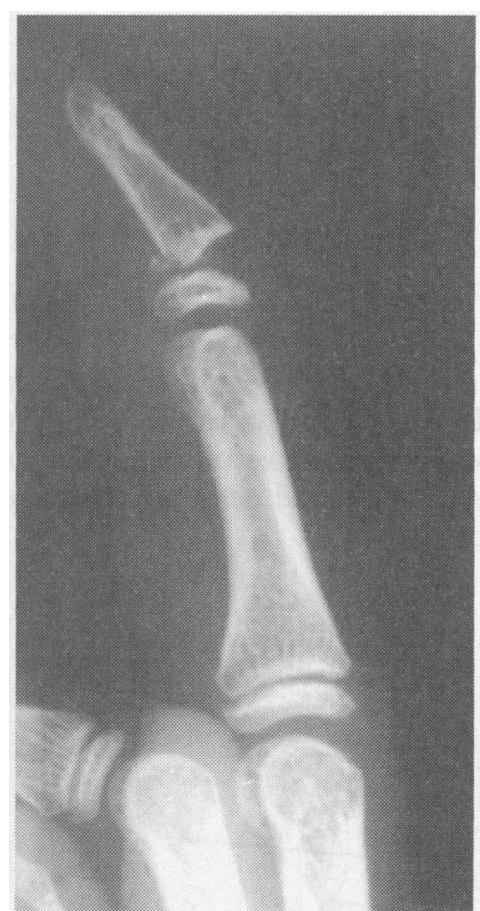

FIG 3-Lateral radiograph of a child's finger showing a type II Salter-Harris epiphyseal injury. There is dorsal displacement of the distal phalanx, and the injury is potentially unstable.
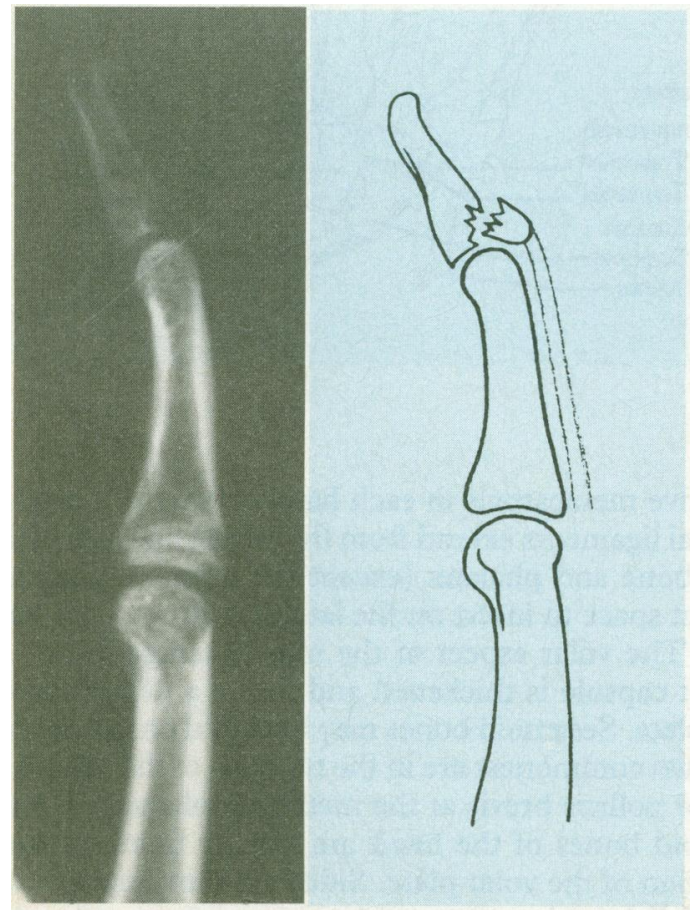

FIG 4-Lateral radiograph of a mallet finger with an avulsion fracture.

\section{Finger tip injuries}

These are commonly produced by a crush injury and may be associated with severe trauma to the soft tissue (including the nail bed and pulp) even if there is no bone injury. Fractures of the distal phalanx in adults and distal phalanx epiphysis in children may be compound into the nail bed. Distal phalanx epiphyseal injuries in children usually displace dorsally affecting the nail bed, and are potentially unstable (fig 3 ).

\section{Mallet finger}

This injury usually results from a direct blow to the extended digit-for example, a blow to the finger tip with a cricket ball. The mallet deformity is produced by avulsion of the extensor tendon from its insertion (not visible on radiography) or by an avulsion fracture at the base of the distal phalanx. Radiography is required to identify a fracture. If the fracture affects more than one third of the joint articular surface the joint may become unstable (fig 4).

\section{Interphalangeal joint dislocation}

This dislocation most commonly affects the proximal interphalangeal joint and is the result of a sporting injury. The volar plate tears or detaches at one end and the distal phalanx usually dislocates dorsally. A joint dislocation may be suspected on the posteroanterior view when the joint space is narrowed or incongruous (fig 5). The diagnosis must be confirmed with the lateral view. In most cases reduction is simple, but occasionally the torn volar plate or other soft tissues become interposed between the joint surfaces and prevent reduction. Radiographs taken after reduction should therefore be checked for possible missed fracture and for perfect joint congruity. Lack of congruity suggests interposition of soft tissue in the joint.

\section{Avulsion fractures}

These are flakes of bone pulled off at the attachments of the ligaments, capsule, or flexor and extensor tendons. They are common and should be suspected from the mechanism of injury and clinical findings. Avulsion flakes can be difficult to show radiologically as they may be visible in only one radiographic projection. The fracture can appear simple, but the underlying soft tissue injury may be serious. The soft tissue swelling visible in the radiograph may be the only indicator of injury.

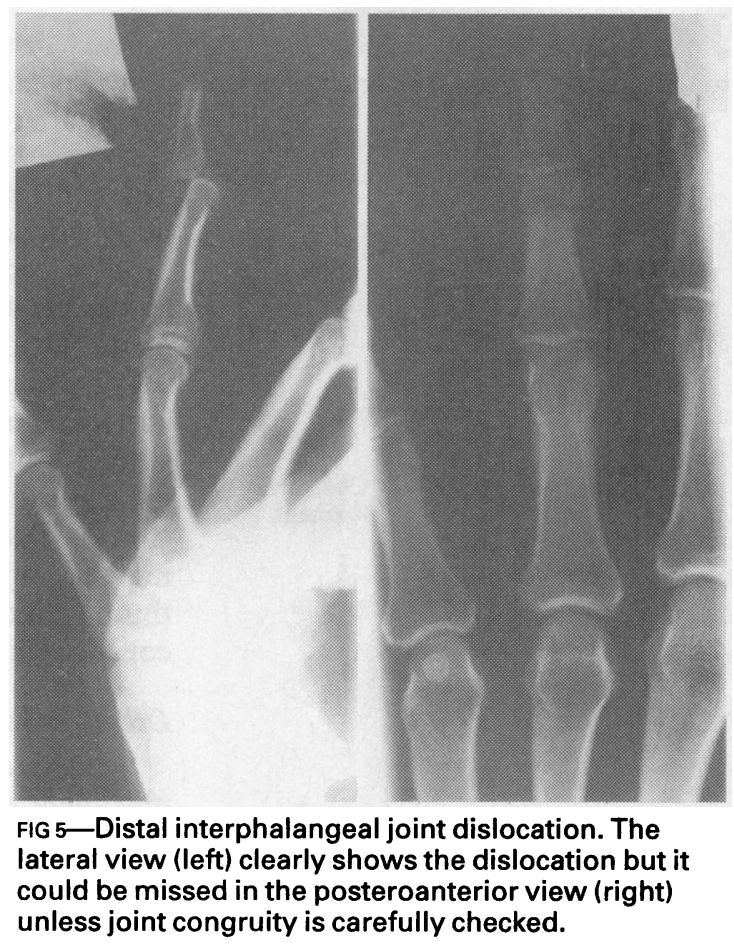




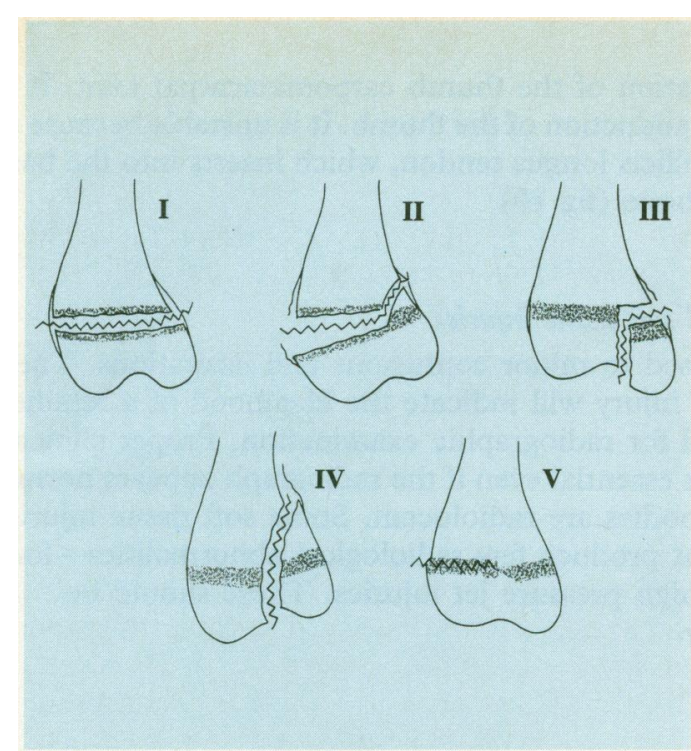

FIG 6-Salter-Harris classification of epiphyseal fractures.

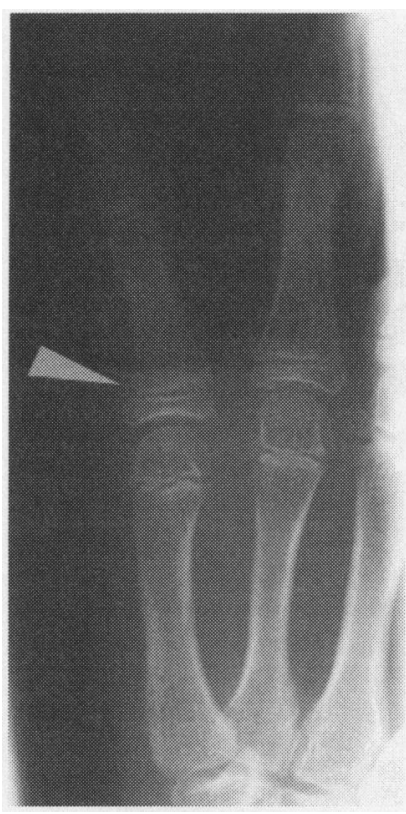

FIG 7-Posteroanterior view of the little finger showing a Salter-Harris type II injury to the base of the proximal phalanx (arrow).

\section{Phalangeal and metacarpal shaft fractures}

The mechanism of injury varies. Transverse and comminuted fractures reflect direct and possibly high energy trauma while spiral fractures are invariably produced by a rotational force. If a fracture is present always check for clinical evidence of rotational deformity in the finger.

Radiological evidence of deformity can be misleading. Oblique radiographs show the metacarpals and phalanges separately. The oblique view is taken close to $90^{\circ}$ to the plane of the posteroanterior radiograph. They should be used for the assessment of these injuries. In the lateral view, which is exactly $90^{\circ}$ to the plane of the posteroanterior radiograph, the metacarpals are superimposed, and this may result in misinterpretation.

\section{Transverse fracture at the base of the proximal phalanx}

This fracture is usually the result of hyperextension of the finger at the metacarpophalangeal joint. Severe dorsal angulation may be present and this can be underestimated clinically and radiologically. An oblique view should be used to assess the injury.

A Salter-Harris type II epiphyseal fracture of the base of the proximal phalanx of the little finger is common in children and results from an abduction force to the finger (fig 7). Ulnar angulation at the fracture site can be seen in the posteroanterior radiograph.

\section{Boxer's fracture}

Fracture of the metacarpal neck with volar displacement of the head usually occurs in the fifth metacarpal bone but it can occur less commonly in the other finger metacarpals (fig 8). It is normally due to a punch. Lateral and oblique views are required to identify any angulation.

Associated wounds to the skin must be taken seriously since they may communicate directly with the metacarpophalangeal joint (particularly after delivering a blow to teeth).

\section{Skier's (gamekeeper's) thumb}

This is an acute sprain or rupture of the ulnar collateral ligament at the metacarpophalangeal joint caused by forceful abduction of the thumb (fig 9). The diagnosis is based on the history and clinical findings. Radiographs can appear normal or show only soft tissue swelling. Occasionally an avulsion fracture may be present.
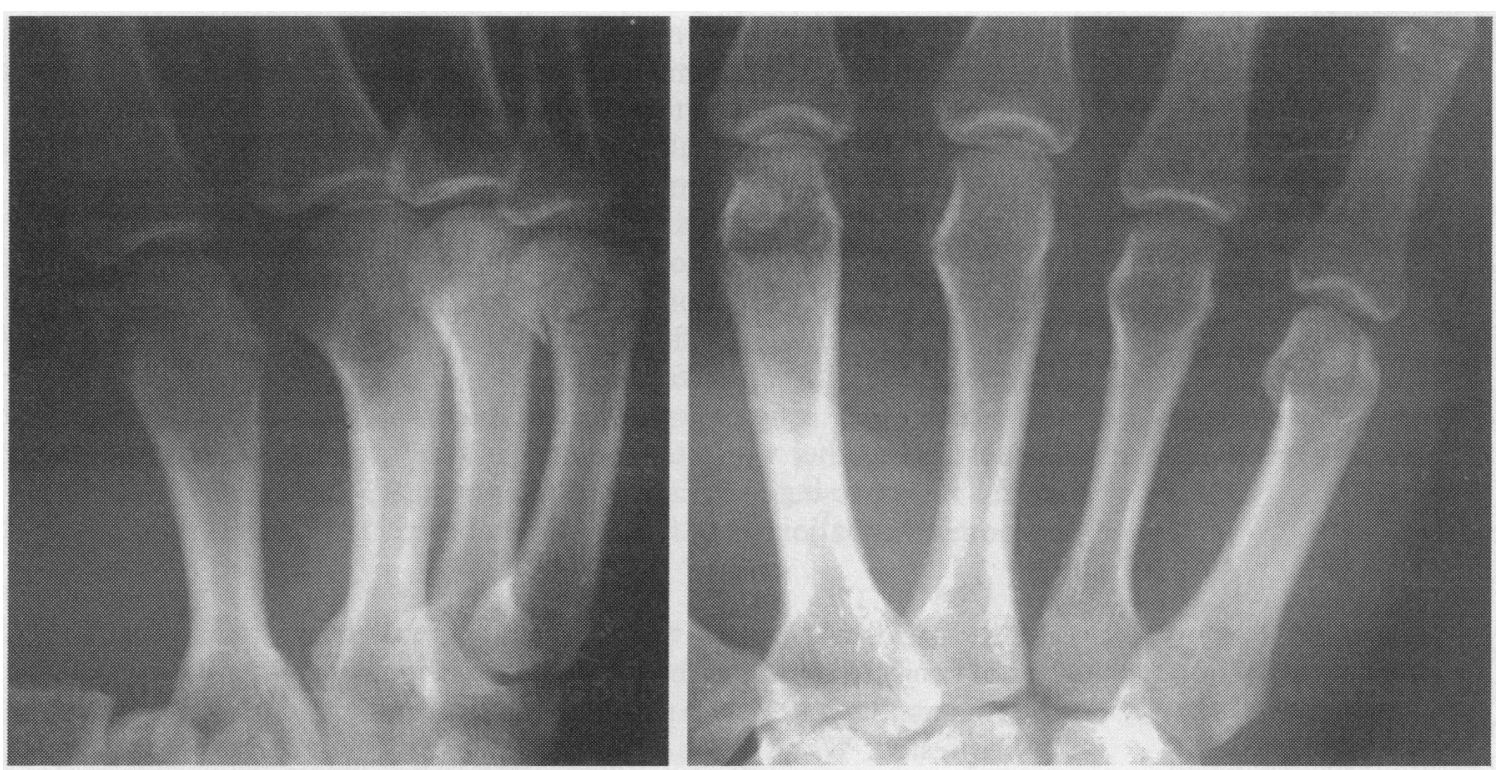

FIG 8-Posteroanterior and oblique view showing a minimally displaced boxer's fracture. 


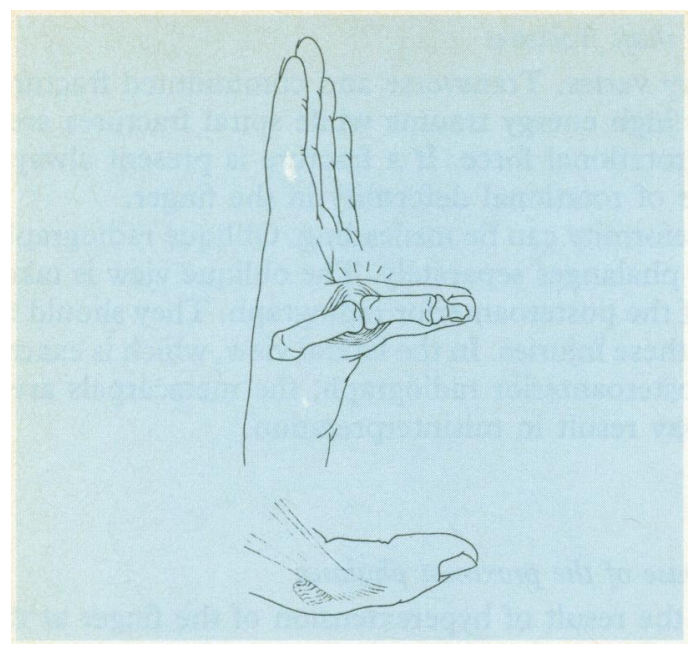

FIG 9-Mechanism of injury in rupture of the ulnar collateral ligament of the thumb

metacarpophalangeal joint. There may be an

avulsion fracture that can be seen in the plain

radiograph; the orientation of the ligament and the

adductor aponeurosis may be identifiable.

\section{Bennett's fracture}

This is a fracture dislocation of the thumb carpometacarpal joint. It usually results from forced abduction of the thumb. It is unstable because of the pull of the abductor pollicis longus tendon, which inserts into the base of the thumb metacarpal bone (fig 10).

\section{Contusions, lacerations, and soft tissue injuries}

The hand is often exposed to minor contusions and lacerations. The history and mechanism of injury will indicate the likelihood of a retained foreign body and the need for radiographic examination. Proper clinical assessment of the wound is essential even if the radiograph appears normal as many retained foreign bodies are radiolucent. Some soft tissue injuries are extremely disabling but produce few radiological abnormalities-for example, pneumatic and high pressure jet injuries. These should be identified from the history.

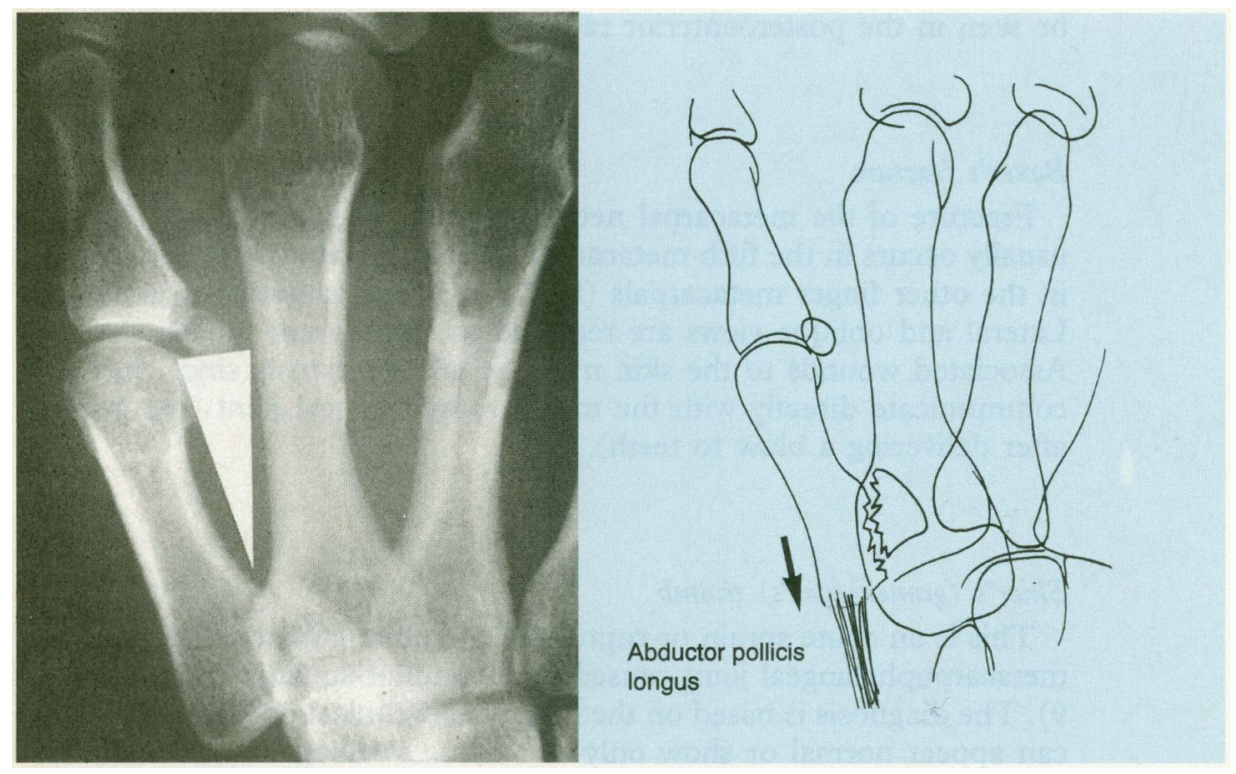

FIG 10-Posteroanterior view and line drawing showing a Bennett's fracture dislocation (arrow).

\section{Radiographic views}

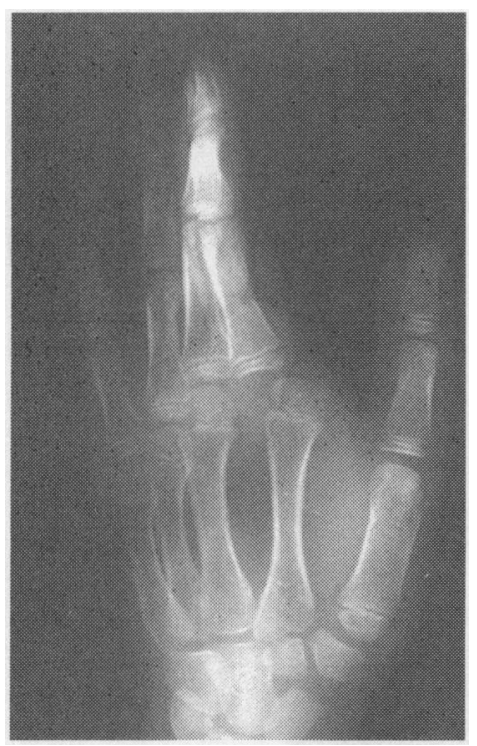

FIG 11-Oblique view of the hand showing a dislocation of the index finger metacarpophalangeal joint.

\section{The hand}

The standard radiographic projections of the whole hand are required to evaluate the base of the proximal phalanges and the metacarpals. When the injury is confined to the distal end of a single digit radiography should be limited to that digit, but the same projections are used.

Posteroanterior view should show the whole of the hand including the base of the metacarpals. It forms the basis of all assessments but is poor at showing fractures of the articular surface of the metacarpal head.

Lateral view-In this projection the metacarpal bones and phalanges are superimposed and can obscure each other. The problem can be partially overcome by flexing the fingers like a fan. The view is essential to show displacement of fracture fragments and joint dislocations.

Posteroanterior oblique view-For complete assessment this view should be requested together with posteroanterior and lateral views. It is particularly useful for assessing dislocation of the metacarpophalangeal and carpometacarpaljointsandfracturesatthebase ofthemetacarpalbones(fig 11).

\section{The thumb}

The standard views of the hand do not give true posteroanterior and lateral projections of the thumb because the plane of the thumb is at $90^{\circ}$ to the fingers. Separate posteroanterior and lateral views of the thumb should be requested. When taken correctly they greatly improve visualisation and recognition of injury at the base of the thumb. 


\section{ABCs system of radiological assessment}

Adequacy

Alignment

Bones

Cartilage

Soft tissues

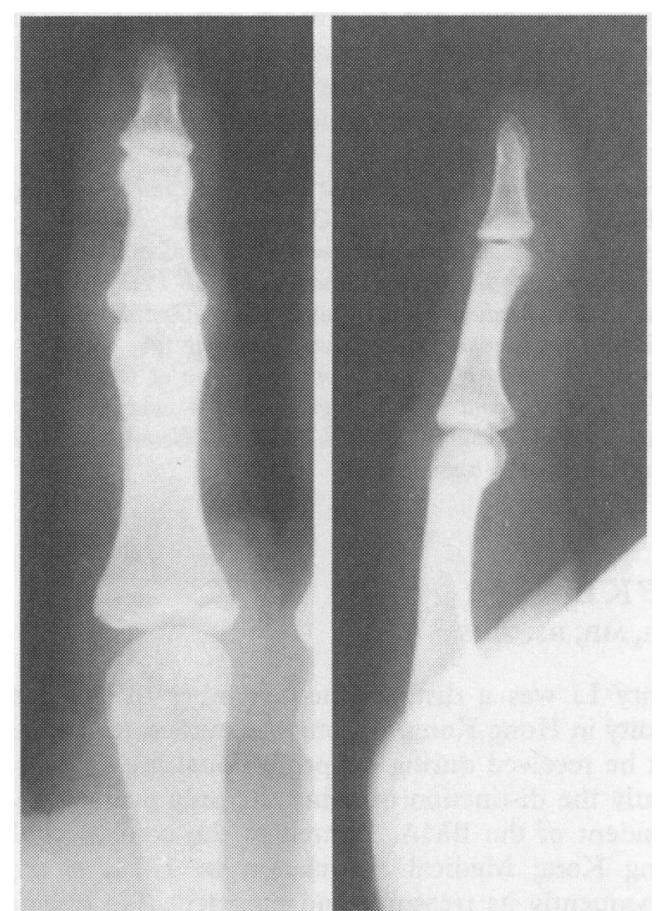

FIG 12-Posteroanterior and lateral view of the index finger. The fracture of the base of the proximal phalanx is visible only in the posteroanterior view. The lateral view is inadequate as it does not show the metacarpophalangeal joint.
Radiographs of the hand should be assessed by using the ABCs system. This system can be applied to all views.

\section{Posteroanterior view}

Check the adequacy and quality of the radiograph

Check alignment of bones-Follow the alignment of the phalanx and metacarpal of each finger and the thumb.
Check bone margins and density - Starting with the thumb and progressing to the fingers, metacarpals, and wrist follow the cortical margin of each bone separately in a clockwise direction. Check the bone density and trabecular pattern as you go. Vascular channels are often seen in the distal shaft of the phalanges and may be mistaken for fractures. They appear as thin, oblique radiolucent lines that run from the external proximal surface obliquely to enter the medullary canal distally.

Check the cartilage and joints-Check each joint space in an orderly fashion, looking specifically at the congruity and separation of the margins of the joint space (fig 5). The bones should not overlap and the joint space should be uniform in width (about $1 \mathrm{~mm}$ ). Compare injured and non-injured joints.

\section{Catches to avoid}

If not true the posteroanterior view may:

- Miss an avulsion fracture affecting the collateral ligament

- Cause soft tissue shadows of the web space to look like a fracture line. The line extends past the cortical margin of the bone and beyond into the soft tissues

If not true the lateral view may miss:

- A joint dislocation

- Avulsion fracture of volar plate

- Fracture through the articular surface of the base of a phalanx

\section{Summary}

History including detailed mechanism of injury

Meticulous clinical examination

Appropriate radiograph and systematic assessment with $A B C$ s system
Check the soft tissues-Soft tissue abnormalities are best seen by using a bright light. Although foreign bodies may be seen in this view, tangential views will remove the superimposed bone shadow and increase the likelihood of visualisation. Soft tissue swelling may be the only indicator of injury.
D W Hodgkinson is senior registrar in emergency medicine, N Kurdy is tutor in orthopaedic surgery, D A Nicholson is consultant radiologist, and P A Driscoll is senior lecturer in emergency medicine, Hope Hospital, Salford.

The line drawings were prepared by Mary Harrison, medical illustrator.

The ABC of Emergency Radiology has been edited by David Nicholson and Peter Driscoll. 